Board of Examiners to agree upon guidelines for circulation to candidates. He suggested that the formulation should always be linked to the clinical situation in which it was to be used, and an examiner should specify this when requesting a formulation. Dr Peter Kennedy (at present a College examiner) was the third speaker. He presented a different viewpoint in that he felt that the formulation should begin with a differential diagnosis and include an account of aetiological factors and psychodynamics.

The ensuing discussion was lively and enthusiastic. One contributor found it hard to believe that candidates were interrupted in the examination if their formulation differed from that used by the examiner and also that they were given no clear expectation as to what was required of them, and it was pointed out that in such a situation the examination became more of a test of the candidate's ability in dealing with an unhelpful examiner.

Another suggestion was that the word formulation should be removed from the examination entirely as the term was ambiguous and a clinical problem could not be adequately 'formulated' after a single conversation with a patient in the absence of any other information. It was commented that a written formulation could be used, such as a letter to the patient's GP in reply to his referral.

Professor Cawley (Chief Examiner) also spoke, warning of the dangers inherent in the reification of formulation. He saw it as a means of bringing a patient alive, and preferred to use the word as a verb- to formulate. The College is beginning to train examiners with the aim of achieving a more uniform approach. He suggested that candidates challenged for not giving an examiner the required reply should discuss the misunderstanding with the examiner and ask him how he defined the term or offer his own 'assessment'. He invited candidates to write to him if they experienced great difficulty.

It was clear that the discussions could have continued for much longer, but there was no shortage of material to feed back to the Examinations Working Party.

Julie A. Hollyman

PHIIP ThOMAs

Collegiate Trainees Committee

\section{Scottish Trainees' Day}

The second Scottish Trainees' Day was held at the Murray Royal Hospital, Perth on 7 April 1983.

Dr Phil Thomas (Royal Edinburgh Hospital) started the day off by introducing us to the proposed changes in the MRCPsych. As far as the Preliminary Test is concerned, the main proposed change is for the introduction of a clinical examination, aimed at testing one's ability to relate to patients. Another suggestion is that after three failures at the MCQ paper, a candidate may be given a 'free shot' at the clinical, but it was felt that it was not unreasonable for a future consultant psychiatrist to pass some multiple choice questions on sciences basic to psychiatry. The proposed changes in format for Part II were thought to be good. It was agreed that the viva should be dropped and replaced by a second 'clinical'. One major point was that we felt that sponsorship had been greatly devalued over the years. Effective sponsorship would be a far better way of assessing one's suitability to become a consultant psychiatrist rather than introducing a clinical examination to the Preliminary Test.

Dr J. D. Templeton (Southern General Hospital) introduced the concept of psychotherapy and the College recommendations for training in psychotherapy at both registrar and senior registrar levels. He emphasized the importance of adequate supervision for any patient taken on for dynamic psychotherapy. It was felt that training in dynamic psychotherapy (with adequate provision) was absolutely essential to anyone intending to become a consultant psychiatrist. Following a proposal from the Collegiate Trainees Committee that it should be a medium term aim that any training post which did not provide psychotherapy supervision were it requested should not be given full approval, a questionnaire on psychotherapy supervision available to trainees was completed by all attending the meeting.

In the morning, Dr D. Brodie (West of Scotland Hospital) talked on the present state of medical unemployment, how it had arisen, and how we might change the situation. Dr Angus McKay (Argyll and Bute Hospitals) discussed training outwith the major centres. As these two topics were strongly linked, they were discussed as one subject. It became clear that very few trainees present could have any certainty of future employment. It was felt that an immediate cut in intake to medical school must be pressed for, and also that there should be expansion of the consultant grade with a relative decrease in the number of junior doctors. This inevitably lead us on to talking about patterns of training, including training in the periphery. There was great concern expressed that if there were to be fewer trainees in psychiatry, that these would be concentrated in the major teaching centres, leaving the periphery with no trainees at all, resulting in decline in stimulation and research with a consequent drop in standards of patient care. It was agreed that the College should cease to recognize individual hospitals for training purposes, and instead should recognize only training schemes which involve rotation to the periphery for a significant period.

The role of the associate specialist was also discussed and it was felt that no new associate specialist posts should be created, and that when their positions became vacant, they should be reviewed and replaced by a training post or a consultant appointment.

The organizers found the day a most rewarding and stimulating experience and would like to thank all those who attended.

Dallas Brodie (Southern General Hospital, Glasgow) LESLIE BuRTON (Crichton Royal Hospital, Dumfries) 